\title{
Performance evaluation considering iterations per phase and SA temperature in WMN-SA system
}

\author{
Shinji Sakamoto ${ }^{\mathrm{a}, *}$, Elis Kulla ${ }^{\mathrm{a}}$, Tetsuya Oda ${ }^{\mathrm{a}}$, Makoto Ikeda ${ }^{\mathrm{b}}$, Leonard Barolli ${ }^{\mathrm{b}}$ and \\ Fatos Xhafa ${ }^{\mathrm{c}}$ \\ ${ }^{a}$ Graduate School of Engineering, Fukuoka Institute of Technology, Fukuoka, Japan \\ ${ }^{\mathrm{b}}$ Department of Information and Communication Engineering, Fukuoka Institute of Technology, \\ Fukuoka, Japan \\ ${ }^{\mathrm{c} T e c h n i c a l ~ U n i v e r s i t y ~ o f ~ C a t a l o n i a, ~ D e p a r t m e n t ~ o f ~ L a n g u a g e s ~ a n d ~ I n f o r m a t i c s ~ S y s t e m s, ~ B a r c e l o n a, ~}$ \\ Spain
}

\begin{abstract}
One of the key advantages of Wireless Mesh Networks (WMNs) is their importance for providing cost-efficient broadband connectivity. There are issues for achieving the network connectivity and user coverage, which are related with the node placement problem. In this work, we consider Simulated Annealing Algorithm (SA) temperature and Iteration per phase for the router node placement problem in WMNs. We want to find the optimal distribution of router nodes in order to provide the best network connectivity and provide the best coverage in a set of Normal distributed clients. From simulation results, we found how to optimize both the size of Giant Component and number of covered mesh clients. When the number of iterations per phase is big, the performance is better in WMN-SA System. From for SA temperature, when SA temperature is 0 and 1 , the performance is almost same. When SA temperature is 2 and 3 or more, the performance decrease because there are many kick ups.
\end{abstract}

Keywords: Wireless Mesh Networks, WMN-SA, giant component, covered mesh clients, iterations per phase, SA temperature, normal distribution

\section{Introduction}

Wireless Mesh Networks (WMNs) [1-3] are important network infrastructure for providing costefficient broadband wireless connectivity. They are showing their applicability in deployment of medical, transport and surveillance applications in urban areas, metropolitan, neighboring communities and municipal area networks. The main issues of WMNs consist of achieving network connectivity and stability as well as QoS in terms of user coverage. These issues are very closely related to the family of node placement problems in WMNs, such as mesh router nodes placement.

Node placement problems have been long investigated in the optimization field due to numerous applications in location science (facility location, logistics, services, etc.) and classification (clustering).

\footnotetext{
${ }^{*}$ Corresponding author: Shinji Sakamoto, Graduate School of Engineering, Fukuoka Institute of Technology (FIT), 3-30-1 Wajiro-Higashi, Higashi-Ku, Fukuoka 811-0295, Japan. E-mail: shinji.t.sakamoto@ gmail.com.
} 


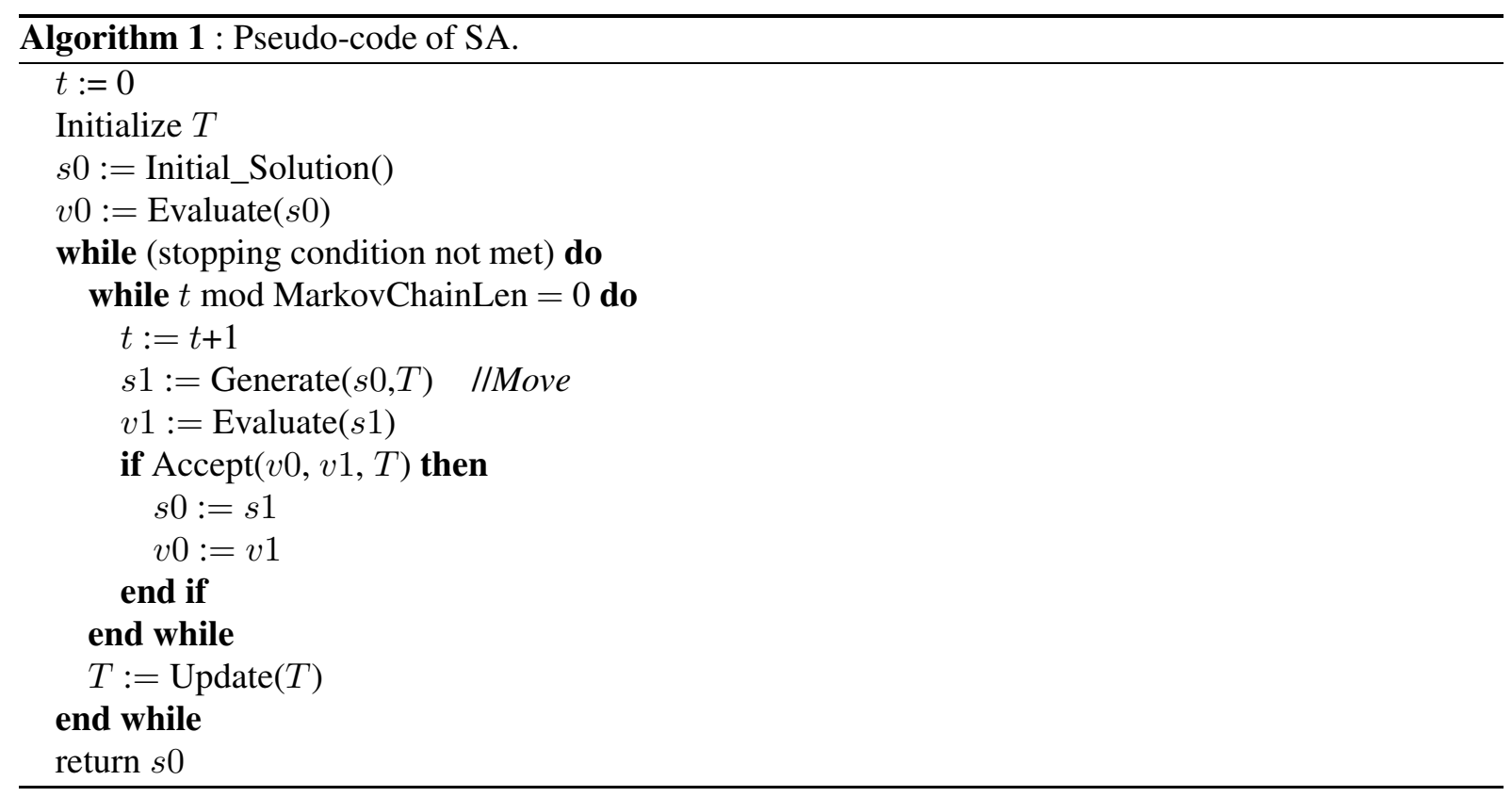

Facility location problems are thus showing their usefulness to communication networks, and more especially from WMNs field. WMNs are currently attracting a lot of attention from wireless research and technology community for providing cost-efficient broadband wireless connectivity.

WMNs are based on mesh topology, in which every node (representing a server) is connected to one or more nodes, enabling thus the information transmission in more than one path. The path redundancy is a robust feature of this kind of topology. Compared to other topologies, mesh topology does not need a central node, allowing networks based on such topology to be self-healing. These characteristics of networks with mesh topology make them very reliable and robust networks to potential server node failures. In WMNs mesh routers provide network connectivity services to mesh client nodes. The good performance and operability of WMNs largely depends on placement of mesh routers nodes in the geographical deployment area to achieve network connectivity, stability and user coverage. The objective is to find an optimal and robust topology of the mesh router nodes to support connectivity services to clients.

For most formulations, node placement problems are shown to be computationally hard to solve to optimality [4-7], and therefore heuristic and meta-heuristic approaches are useful approaches to solve the problem for practical purposes. Several heuristic approaches are found in the literature for node placement problems in WMNs [8-12].

In this work, we use our proposed and implemented WMN-SA system, which is based on Simulation Annealing (SA) to deal with the node placement problem in WMNs. For simulations, we consider normal distribution of 48 mesh clients in a $32 \times 32$ grid size. Then we deploy 16 mesh routers and apply $\mathrm{SA}$, to maximize the size of Giant Component (GC) and then maximize the number of covered mesh clients.

The rest of the paper is organized as follows. The definition of node placement problem is presented in Section 2. The proposed and implemented WMN-SA simulation system is presented in Section 3. The simulation results are given in Section 4. Finally, concluding remarks and future work are given in Section 5. 


\section{Node placement problem in WMNs}

In this problem, we are given a grid area arranged in cells where to distribute a number of mesh router nodes and a number of mesh client nodes of fixed positions (of an arbitrary distribution) in the grid area. The objective is to find a location assignment for the mesh routers to the cells of the grid area that maximizes the network connectivity and client coverage. Network connectivity is measured by the size of the GC of the resulting WMN graph, while the user coverage is simply the number of mesh client nodes that fall within the radio coverage of at least one mesh router node.

An instance of the problem consists as follows.

- $N$ mesh router nodes, each having its own radio coverage, defining thus a vector of routers.

- An area $W \times H$ where to distribute $N$ mesh routers. Positions of mesh routers are not predetermined, and are to be computed.

- $M$ client mesh nodes located in arbitrary points of the considered area, defining a matrix of clients.

It should be noted that network connectivity and user coverage are among most important metrics in WMNs and directly affect the network performance.

In this work, we have considered a bi-objective optimization in which we first maximize the network connectivity of the WMN (through the maximization of the size of the GC) and then, the maximization of the number of covered mesh clients.

\section{Proposed and implemented WMN-SA system}

In this section, we present WMN-SA simulation system. Our system can generate instances of the problem using different distributions of client and mesh routers.

The GUI of WMN-SA is shown in Fig. 1. We set the network configuration parameters as number of clients, client distribution, number of mesh routers, grid size, radius of communication distance and the size of subgrid.

\subsection{Simulated annealing}

\subsubsection{Description of simulated annealing}

SA algorithm [13] is a generalization of the metropolis heuristic. Indeed, SA consists of a sequence of executions of metropolis with a progressive decrement of the temperature starting from a rather high temperature, where almost any move is accepted, to a low temperature, where the search resembles Hill Climbing. In fact, it can be seen as a hill-climber with an internal mechanism to escape local optima (see pseudo-code in Algorithm 1). In SA, the solution $s^{\prime}$ is accepted as the new current solution if $\delta \leqslant 0$ holds, where $\delta=f\left(s^{\prime}\right)-f(s)$. To allow escaping from a local optimum, the movements that increase the energy function are accepted with a decreasing probability $\exp (-\delta / T)$ if $\delta>0$, where $T$ is a parameter called the "temperature". The decreasing values of T are controlled by a cooling schedule, which specifies the temperature values at each stage of the algorithm. This represents an important decision for its application (a typical option is to use a proportional method, like $T_{k}=\alpha \cdot T_{k-1}$ ). SA usually gives better results in practice, but uses is very slow. The most striking difficulty in applying SA is to choose and tune its parameters such as initial and final temperature, decrement of the temperature (cooling schedule), equilibrium detection, etc. 


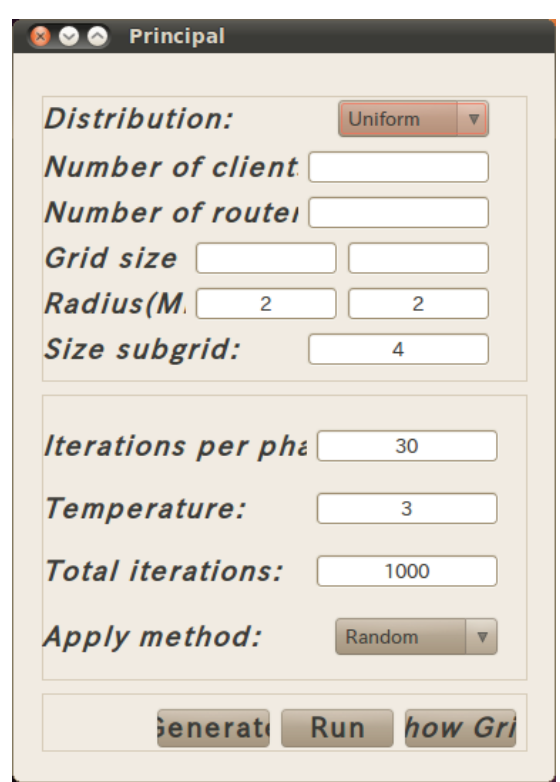

Fig. 1. GUI tool for WMN-SA system.

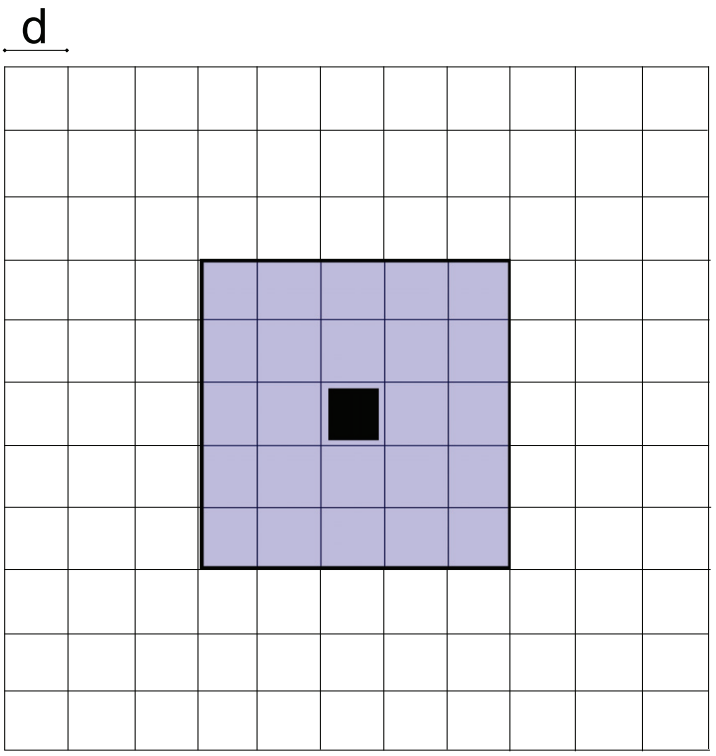

Fig. 2. Mesh router coverage and communication distance.

For further details on initial solution, fitness evaluation and movement types, refer to [14] ${ }^{1}$. However, the acceptability criteria of neighboring solutions is now different, as explained next.

\subsubsection{Acceptability criteria}

The acceptability criteria for newly generated solutions is based on the definition of a threshold value (accepting threshold) as follows. We consider a succession $t_{k}$ such that $t_{k}>t_{k+1}, t_{k}>0$ and $t_{k}$ tends to 0 as $k$ tends to infinity. Then, for any two solutions $s_{i}$ and $s_{j}$, if fitness $\left(s_{j}\right)-$ fitness $\left(s_{i}\right)<t_{k}$, then accept solution $s_{j}$.

For the SA, $t_{k}$ values are taken as accepting threshold but the criterion for acceptance is probabilistic:

- If fitness $\left(s_{j}\right)$ - fitness $\left(s_{i}\right) \leqslant 0$ then $s_{j}$ is accepted.

- If fitness $\left(s_{j}\right)$ - fitness $\left(s_{i}\right)>0$ then $s_{j}$ is accepted with probability $\exp \left[\left(f i t n e s s\left(s_{j}\right)\right.\right.$-fitness $\left.\left.\left(s_{i}\right)\right) / t_{k}\right]$ (at iteration $k$ the algorithm generates a random number $R \in(0,1)$ and $s_{j}$ is accepted if $R<$ $\exp \left[\left(\right.\right.$ fitness $\left(s_{j}\right)-$ fitness $\left.\left.\left.\left(s_{i}\right)\right) / t_{k}\right]\right)$.

In this case, each neighbor of a solution has a positive probability of replacing the current solution. The $t_{k}$ values are chosen in a way that solutions with large increase in the cost of the solutions are less likely to be accepted (but there is still a positive probability of accepting them).

\section{Simulation results}

\subsection{Simulation settings}

We carried out many simulations to evaluate the performance of WMNs using WMN-SA simulation system. In these simulation scenarios, we consider a grid with $32 \times 32$ size. One grid unit is $20 \mathrm{~m} \times 20 \mathrm{~m}$

\footnotetext{
${ }^{1}$ Initial solution, fitness evaluation and movement types are the same for Hill Climbing and Simulated Annealing.
} 
Table 1

Simulation settings

\begin{tabular}{lc}
\hline Parameters & Values \\
\hline Client distribution & Normal \\
Area size & $32 \times 32$ \\
Number of mesh router nodes & 16 \\
Number of mesh client nodes & 48 \\
SA temperature & $0,1,2,3$ \\
Iteration per phase & $64,128,256,512$ \\
Applied method & Combination \\
\hline
\end{tabular}

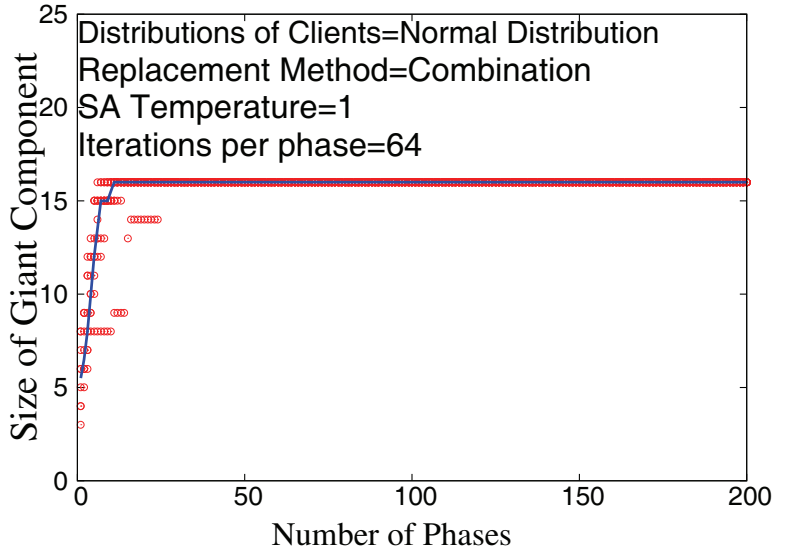

(a) Iterations per phase $=64$

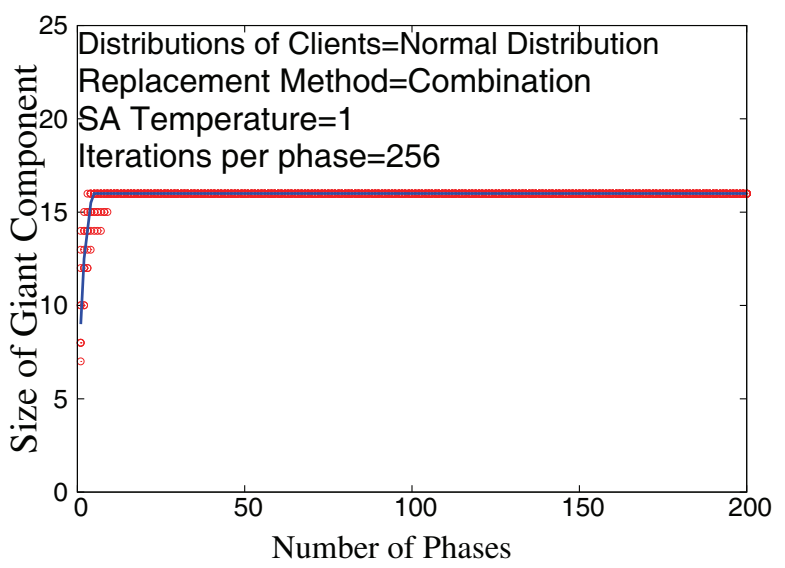

(c) Iterations per phase $=256$

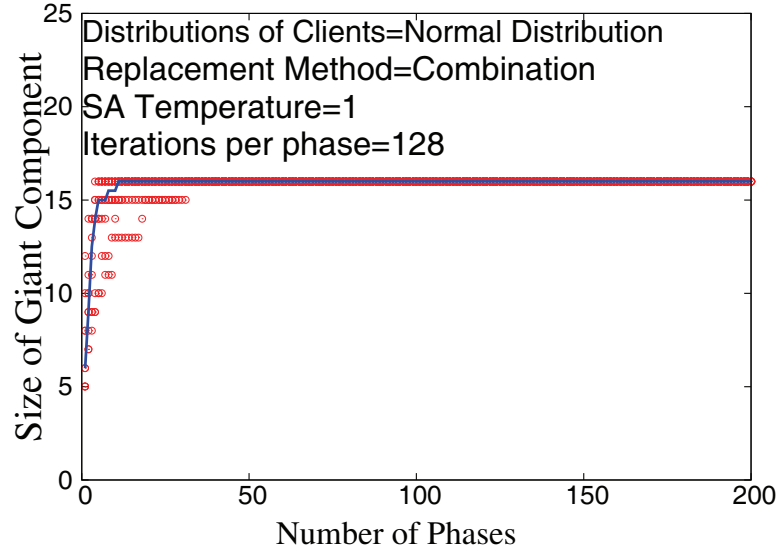

(b) Iterations per phase $=128$

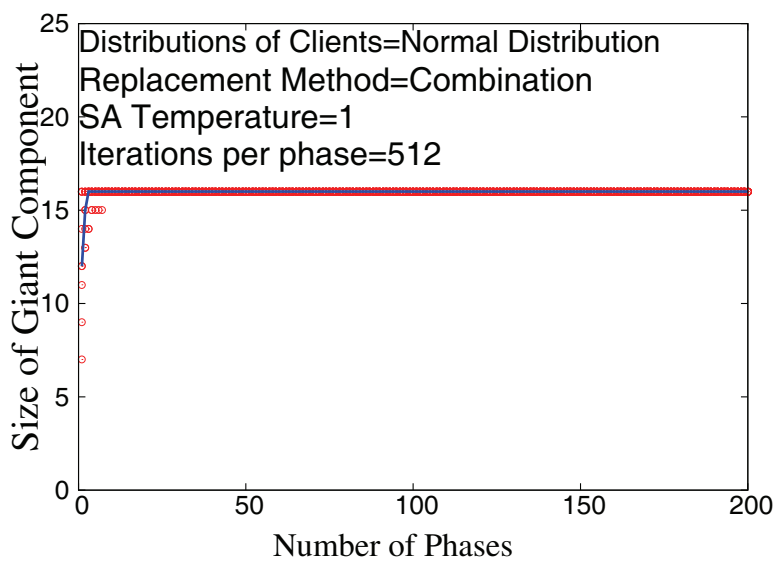

(d) Iterations per phase $=512$

Fig. 3. Size of GC for different iterations per phase.

$(d=20 \mathrm{~m})$. In Fig. 2, we show the coverage area of a mesh router, which has a radius of $50 \mathrm{~m}$. In Table 1, we show the simulation parameters. The distribution of mesh clients is normal. The number of mesh routers is 16 and the number of mesh clients 48 . For evaluation, we considered 4 different values of iterations per phase $(64,128,256,512)$ and SA temperature $(0,1,2,3)$. It should be noted that when the value of temperature is larger or equal to 1 , the SA may accept sometimes bad solutions. 


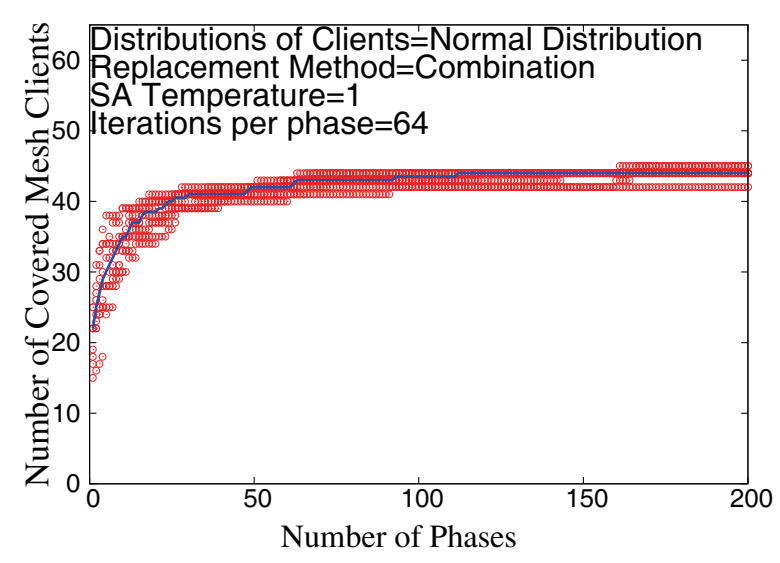

(a) Iterations per phase $=64$

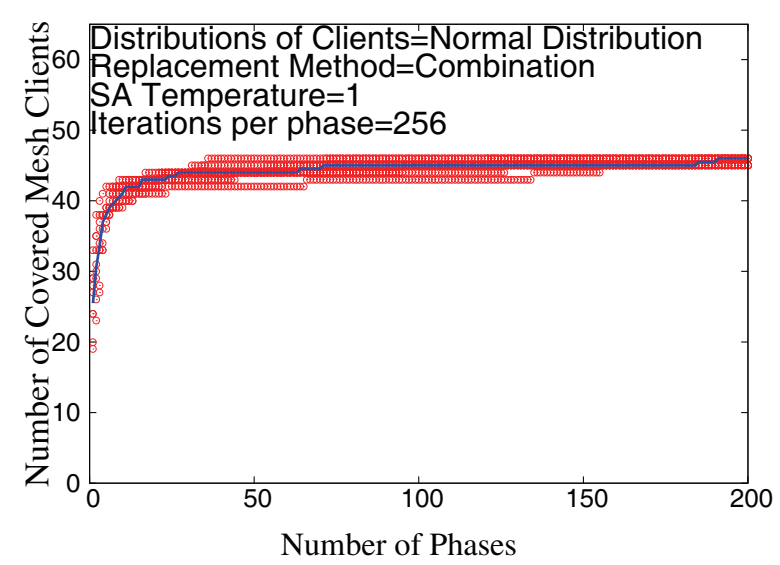

(c) Iterations per phase $=256$

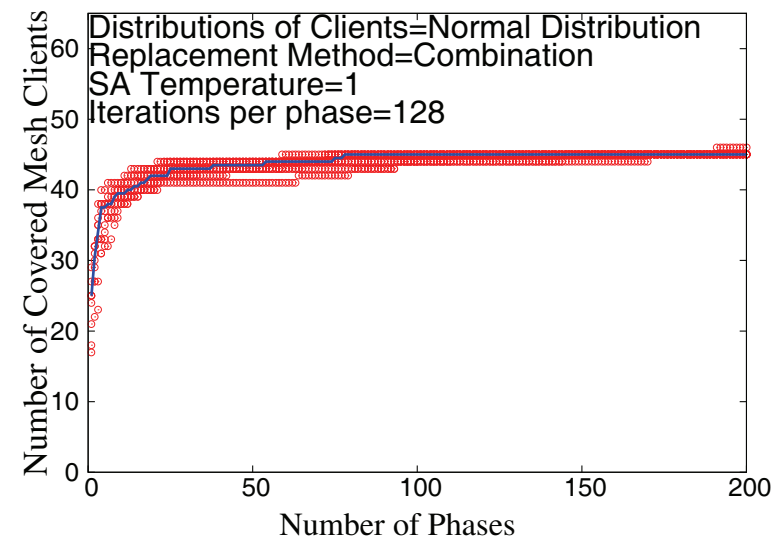

(b) Iterations per phase $=128$

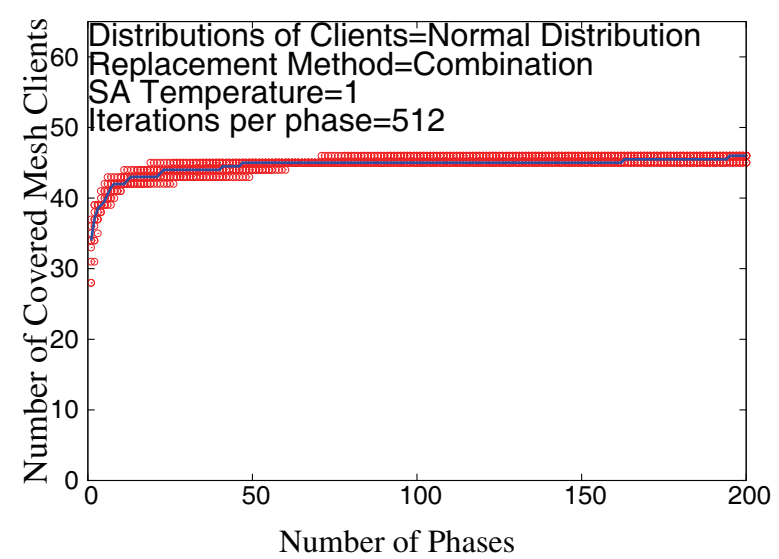

(d) Iterations per phase $=512$

Fig. 4. Number of covered mesh clients for different iterations per phase.

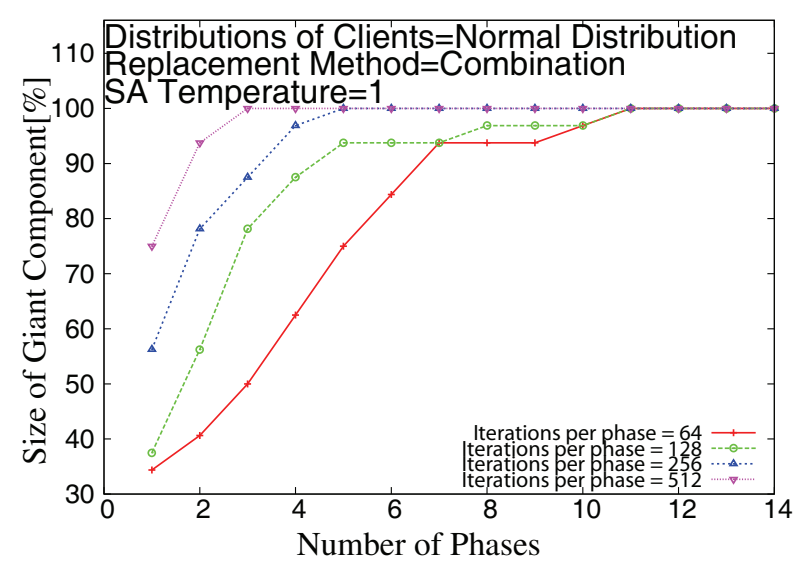

(a) Size of GC

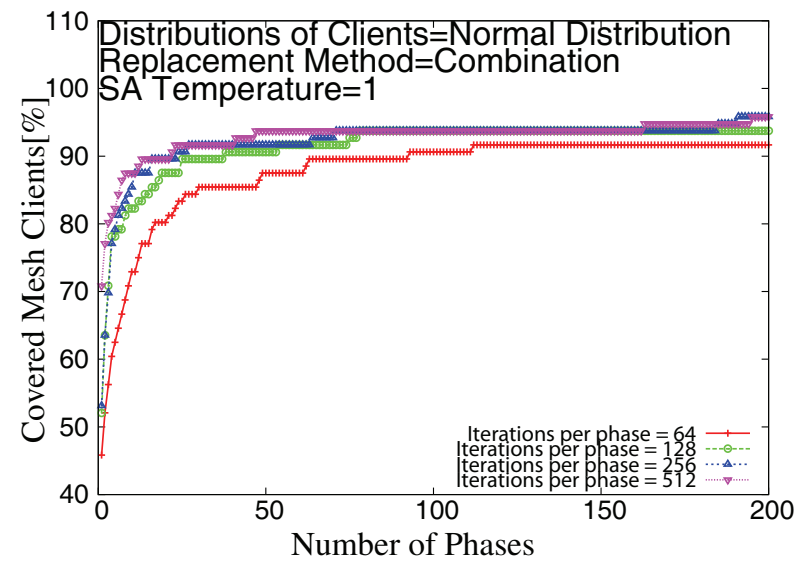

(b) Number of covered mesh clients

Fig. 5. Comparison of 4 different iterations per phase. 


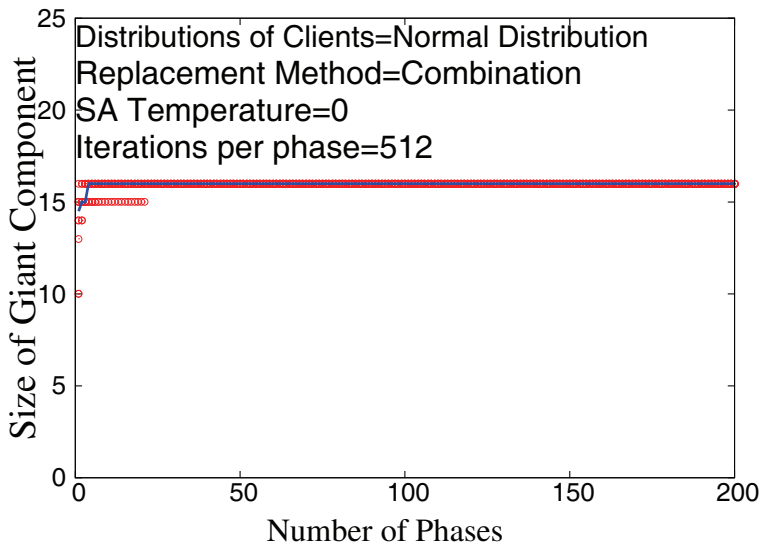

(a) SA temperature $=0$

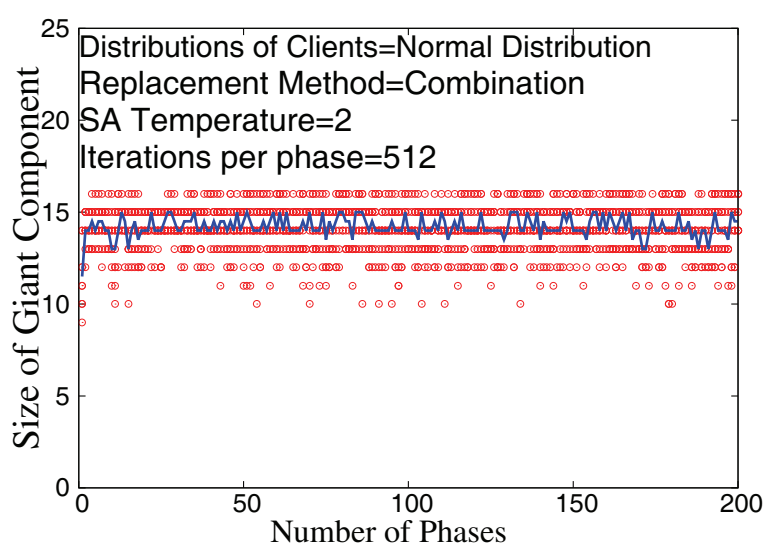

(c) SA temperature $=2$

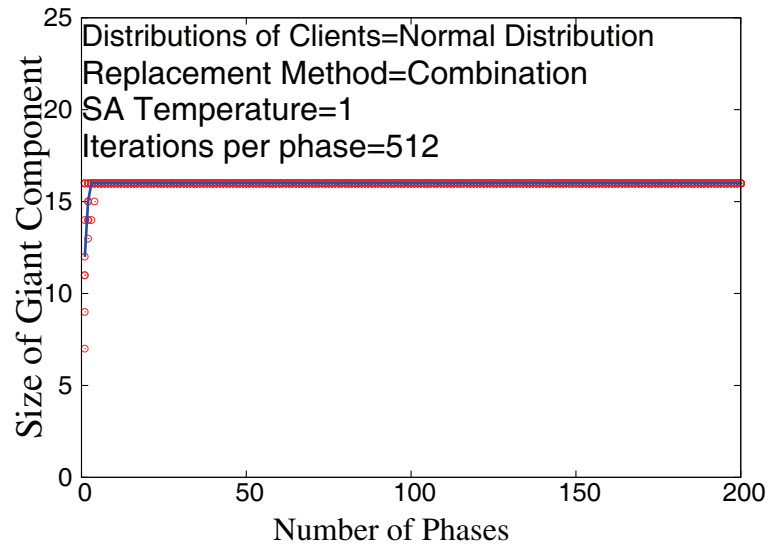

(b) SA temperature $=1$

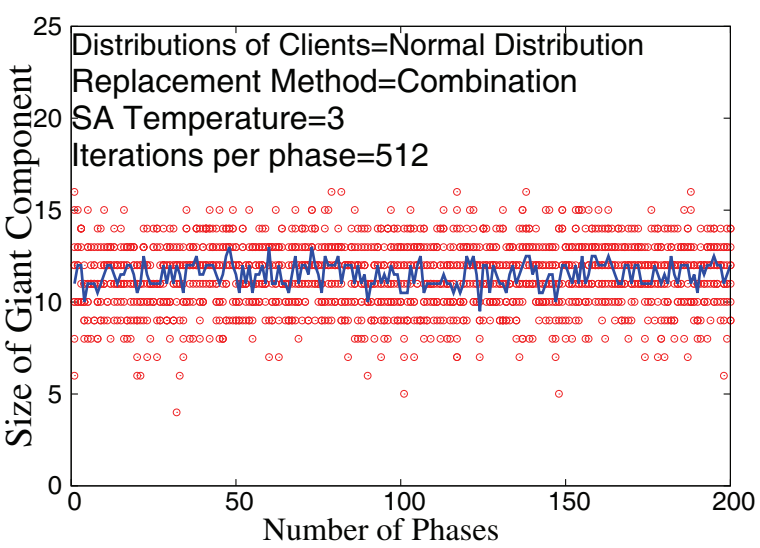

(d) SA temperature $=3$

Fig. 6. Size of GC for different SA temperature.

\subsection{Results discussion}

In Figs 3, 4, 6 and 7, we show results of size of GC and number of covered mesh clients. For each phase of calculations, we conduct the simulations 10 times, in order to create a general view of results. The maximum size of GC is 16 , as we have 16 routers in our scenario.

\subsubsection{Evaluation for different iterations per phase}

We conduct simulations for different number of iterations per each phase.

In Figs 3 and 4, we show results of size of GC and number of covered mesh clients, respectively. For each phase of calculations, SA runs a number of $64,128,256$ and 512 iterations.

In Figs 3(a) and 3(b), the performance of the system for optimizing the size of GC, is almost the same. After 25 iterations the router backbone is completed with all 16 routers. While in the cases of 256 and 512 iterations (Figs 3(c) and 3(d)), the performance increases, as the max size of GC is reached for less phases (10 and 8, respectively). In Fig. 5(a), we show the results of the size of GC up to 14 phases as the size of GC is always maximum for more phases.

In Fig. 4 are shown the results for number of covered mesh clients. We conducted simulations with 200 phases. The algorithm runs in similar ways for all cases when number of iterations is different. The 


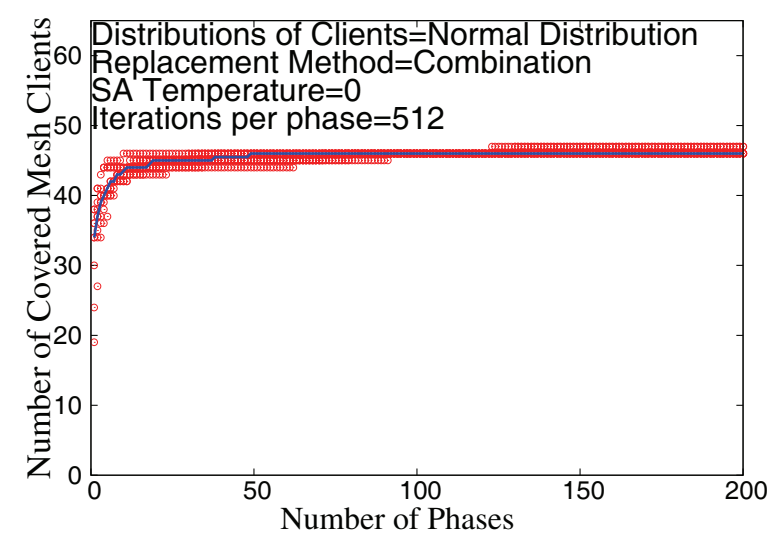

(a) SA temperature $=0$

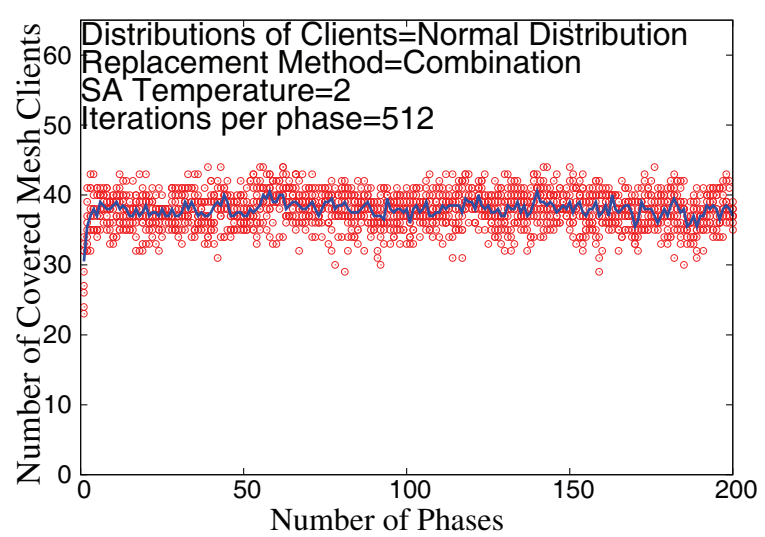

(c) SA temperature $=2$

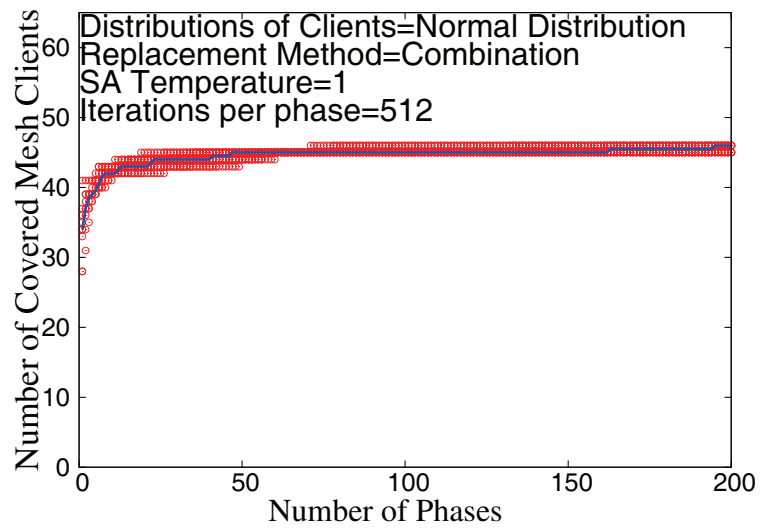

(b) SA temperature $=1$

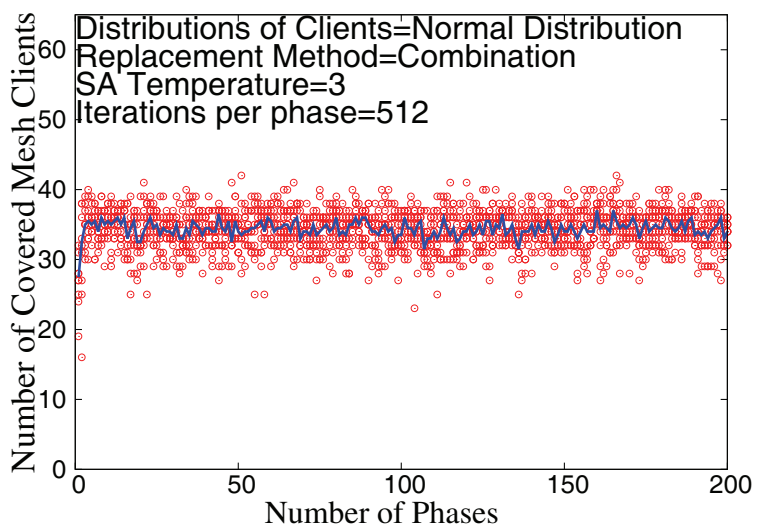

(d) SA temperature $=3$

Fig. 7. Number of covered mesh clients for different SA temperature.

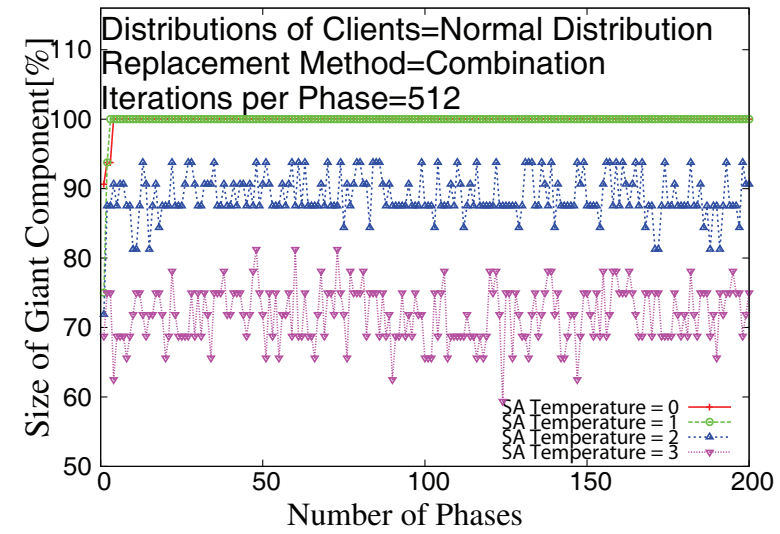

(a) Ratios of Giant Component

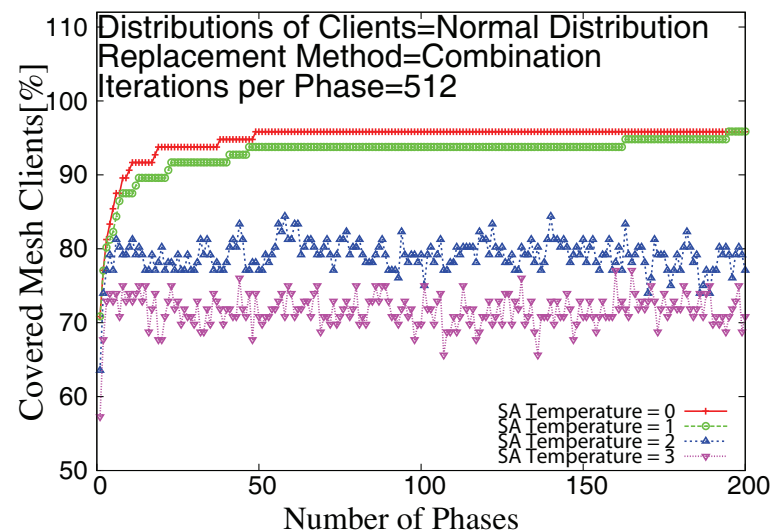

(b) Ratios of fovered mesh clients

Fig. 8. Comparison for different SA temperature. 
maximum number of covered mesh clients goes up to 46 . The performance becomes a little better when we move from 64 iterations per phase up to 512 (see Figs 4(a)-4(d)), in terms of faster optimization. In the cases of 256 and 512 iterations per phase, in Figs 4(c) and 4(d), in around 10 phases there are more than 40 mesh clients covered. In Fig. 5(b), for less iterations per phase the rate of covered mesh clients is smaller.

\subsubsection{Evaluation for different SA temperature values}

We conduct tests for different values of SA temperature (from 0 to 3), while keeping the number of iterations per phase fixed to 512 . We should note that when the value of temperature is $0, \mathrm{SA}$ is a simple $\mathrm{HC}$ and there is no tolerance for finding bad solutions.

In Figs 6 and 7, we show results of size of GC and number of covered mesh clients, respectively. For 200 phases of calculations, when SA temperature is 0 and 1 (Figs 6(a) and 6(b)), the performance is good and the maximum size of GC is reached in a few phases. In Figs 6(c) and 6(d), the SA temperature is increased to values 2 and 3 . We notice that, because of many kick-ups of SA, the performance decreases and the max size of GC (16) is not reached in most of the cases. The average size of GC is smaller when temperature value is 3 .

In Fig. 7 is shown the number of covered mesh clients for each SA temperature values. The total number of deployed mesh clients is 48 and in the first two cases (Figs 7(a) and 7(b)), the number of covered mesh clients reaches 47 and 46 , respectively. The performance decreases further to 44 and 41 when temperature increases to 2 and 3, respectively (see Figs 7(c) and 7(d)).

\section{Conclusions}

In this paper, we conducted simulations with our WMN-SA system, in a grid with size $32 \times 32$, where we deployed 48 mesh clients and 16 mesh routers. Using SA, we optimized the size of GC and then the number of covered mesh clients for different iterations per phase and values of SA temperature.

From the simulation results, we conclude that, when we use SA, the performance is better when the number of iterations per phase is bigger. While, when the temperature of SA increases, the performance decreases for both sizes of GC and number of covered mesh clients.

In our future work, we would like to make evaluations for different cases and patterns. Moreover, we would like to implement other search optimization algorithms in our simulation system.

\section{Acknowledgment}

This work is support by a Grant-in-Aid for scientific research of Japanese Society for the Promotion of Science (JSPS). The authors would like to thank JSPS for the financial support.

\section{References}

[1] I.F. Akyildiz, X. Wang and W. Wang, Wireless Mesh Networks: A Survey, Computer Networks 47(4), 2005, $445-487$.

[2] N. Nandiraju, D. Nandiraju, L. Santhanama, B. He, J. Wang and D. Agrawal, Wireless Mesh Networks: Current Challenges and Future Direction of Web-in-the-Sky, IEEE Wireless Communications (2007), 79-89.

[3] Ch. Chen and Ch. Chekuri, Urban Wireless Mesh Network Planning: The Case of Directional Antennas, Tech Report No. UIUCDCS-R-2007-2874, Department of Computer Science, University of Illinois at Urbana-Champaign, 2007. 
[4] M.R. Garey and D.S. Johnson, Computers and Intractability -A Guide to the Theory of NP-Completeness, Freeman, San Francisco, 1979.

[5] A. Lim, B. Rodrigues, F. Wang and Zh. Xua, $k$-Center Problems with Minimum Coverage, Theoretical Computer Science 332(1-3) (2005), 1-17.

[6] E. Amaldi, A. Capone, M. Cesana, I. Filippini and F. Malucelli, Optimization Models and Methods for Planning Wireless Mesh Networks, Computer Networks 52 (2008), 2159-2171.

[7] J. Wang, B. Xie, K. Cai and D.P. Agrawal, Efficient Mesh Router Placement in Wireless Mesh Networks, MASS-2007, Pisa, Italy, 2007, pp. 9-11.

[8] S.N. Muthaiah and C. Rosenberg, Single Gateway Placement in Wireless Mesh Networks, In Proc. of 8th International IEEE Symposium on Computer Networks, Turkey, 2008, pp. 4754-4759.

[9] P. Zhou, B.S. Manoj and R. Rao, A Gateway Placement Algorithm in Wireless Mesh Networks, Proc. of the Third Annual International Wireless Internet Conference (WICON-2007), October 2007, pp. 1-9.

[10] M. Tang, Gateways Placement in Backbone Wireless Mesh Networks, International Journal of Communications, Network and System Sciences 2(1) (2009), 45-50.

[11] A. Antony Franklin and C. Siva Ram Murthy, Node Placement Algorithm for Deployment of Two-Tier Wireless Mesh Networks, In Proc. of IEEE GLOBECOM-2007, Washington, USA, 2007, pp. 4823-4827.

[12] T. Vanhatupa, M. Hännikäinen and T.D. Hämäläinen, Genetic Algorithm to Optimize Node Placement and Configuration for WLAN Planning, In Proc. of 4th International Symposium on Wireless Communication Systems, 2007, pp. 612-616.

[13] S. Kirkpatrick, C.D. Gelatt and M.P. Vecchi, Optimization by Simulated Annealing, Journal of Science 220 (1983), 671-680.

[14] F. Xhafa, C. Sanchez, L. Barolli and R. Miho, An Annealing Approach to Router Nodes Placement Problem in Wireless Mesh Networks, In Proc. of CISIS-2010, 2010, pp. 245-252.

[15] J. Holland, Adaptation in Natural and Artificial Systems, University of Michigan Press, Ann Arbor, 1975.

[16] F. Xhafa, C. Sanchez and L. Barolli, Genetic Algorithms for Efficient Placement of Router Nodes in Wireless Mesh Networks, Proc. of AINA-2010, 2010, pp. 465-472. 

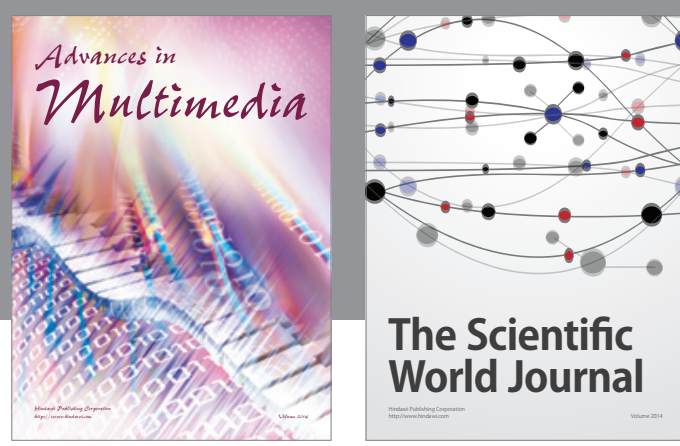

The Scientific World Journal
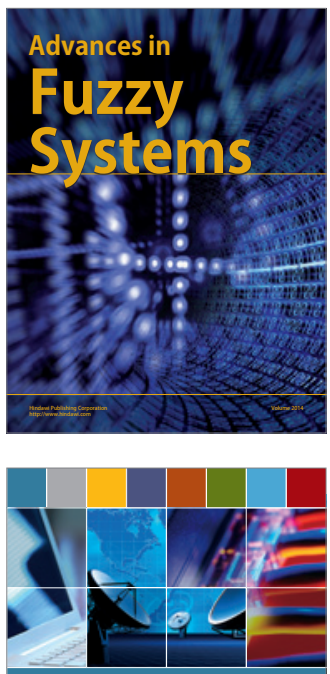

Computer Networks and Communications
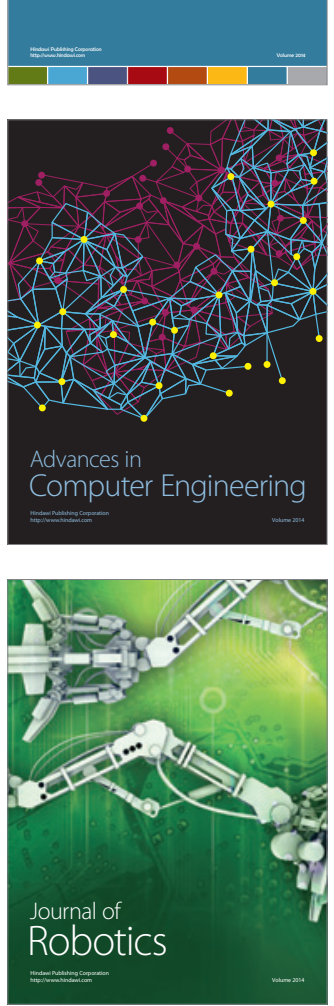
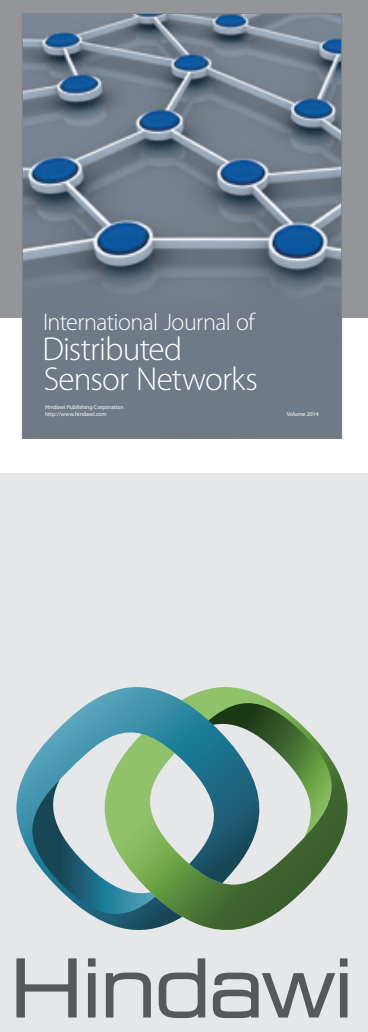

Submit your manuscripts at

http://www.hindawi.com
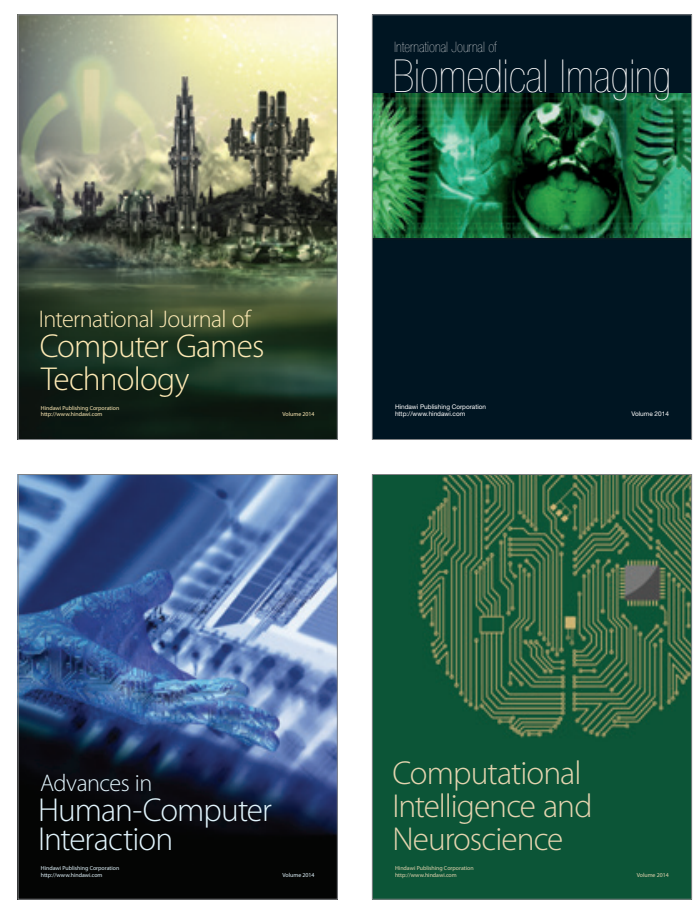
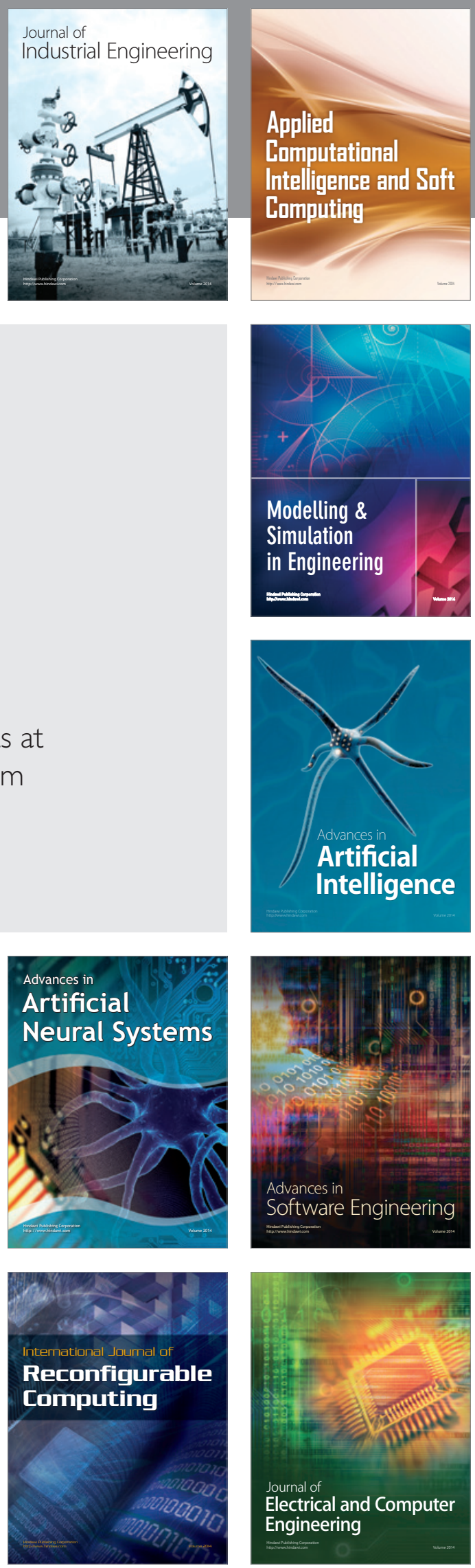